\title{
Rapid necrotic killing of polymorphonuclear leukocytes is caused by quorum-sensing- controlled production of rhamnolipid by Pseudomonas aeruginosa
}

\author{
Peter Ø. Jensen, ${ }^{1}$ Thomas Bjarnsholt, ${ }^{2}$ Richard Phipps, ${ }^{2}$ \\ Thomas B. Rasmussen, ${ }^{2}$ Henrik Calum, ${ }^{1}$ Lars Christoffersen, ${ }^{1}$ \\ Claus Moser, ${ }^{1}$ Paul Williams, ${ }^{4}$ Tacjana Pressler, ${ }^{3}$ Michael Givskov ${ }^{2}$ \\ and Niels Høiby ${ }^{1}$ \\ ${ }^{1}$ Department of Clinical Microbiology, Rigshospitalet, DK-2100 Copenhagen $\varnothing$, Denmark \\ ${ }^{2}$ Centre for Biomedical Microbiology, BioCentrum, Technical University of Denmark \\ ${ }^{3}$ Copenhagen CF Center, Rigshospitalet, DK-2100 Copenhagen $\varnothing$, Denmark \\ ${ }^{4}$ Centre for Biomolecular Sciences, University of Nottingham, UK
}

Correspondence

Niels Høiby

Hoiby@inet.uni2.dk

Received 30 October 2006

Revised 15 December 2006

Accepted 23 December 2006

\begin{abstract}
Quorum sensing (QS) denotes a density-dependent mode of inter-bacterial communication based on signal transmitter molecules. Active QS is present during chronic infections with the opportunistic pathogen Pseudomonas aeruginosa in immunocompromised patients. The authors have previously demonstrated a QS-regulated tolerance of biofilm bacteria to the antimicrobial properties of polymorphonuclear leukocytes (PMNs). The precise QS-regulated effect on the PMNs is, however, unknown. Incubation of human PMNs with supernatants from dense $P$. aeruginosa cultures showed that the QS-competent $P$. aeruginosa induced rapid necrosis of the PMNs. This mechanism was also observed in mouse lungs infected with $P$. aeruginosa, and in sputum obtained from $P$.-aeruginosa-infected patients with cystic fibrosis. Evidence is presented that the necrotic effect was caused by rhamnolipids, production of which is aS controlled. The results demonstrate the potential of the QS system to facilitate infections with $P$. aeruginosa by disabling the PMNs, which are a major first line of defence of the host. Furthermore, the study emphasizes the inhibition of QS as a target for the treatment of infections with $P$. aeruginosa.
\end{abstract}

\section{INTRODUCTION}

Pseudomonas aeruginosa is an opportunistic human pathogen, uncommon as a natural flora, but infecting hospitalized and immunocompromised patients, such as burn victims and AIDS patients. In particular, in patients with the autosomal recessive disease cystic fibrosis $(\mathrm{CF})$, chronic $P$. aeruginosa lung infections represent the major morbid complication (Koch \& Høiby, 1993). The lung infection experienced by CF patients can be divided into two stages: the colonizing non-mucoid state, and the chronic mucoid

\footnotetext{
Abbreviations: $\mathrm{AHL}, \mathrm{N}$-acylhomoserine lactone; BAL, broncheoalveolar lavage; CF, cystic fibrosis; C4-HSL, N-butanoyl-L-homoserine lactone; fMLP, $N$-formyl-L-methionyl-L-leucyl-L-phenylalanine; 3-oxo-C12-HSL, $\mathrm{N}$-3-oxododecanoyl-L-homoserine lactone; $\mathrm{Pl}$, propidium iodide; $\mathrm{PMN}$, polymorphonuclear leukocyte; PQS, Pseudomonas quinolone signal; OS, quorum sensing; 4Q, 4-quinolone.

A time-lapse movie showing the rapid death and disintegration of PMNs is available as supplementary data with the online version of this paper.
}

state. The chronic infection is preceded by intermittent colonizations and infections by non-mucoid $P$. aeruginosa for a mean period of 12 months (Høiby, 1974; Johansen \& Høiby, 1992). During this stage, $P$. aeruginosa is apparently cleared. The gradual transition from the colonizing state to the chronic infection is characterized by a phenotypical switch from the non-mucoid to the mucoid state. This may be caused primarily by $\mathrm{H}_{2} \mathrm{O}_{2}$ liberated by the polymorphonuclear leukocyte (PMNs) (Mathee et al., 1999), and, as recently demonstrated, by anaerobiosis of $P$. aeruginosa in the mucopurulent masses of the bronchioles (Worlitzsch et al., 2002). The presence of $P$. aeruginosa growing in biofilms, i.e. microcolonies surrounded by a self-made polysaccharide matrix, is a hallmark of the chronic lung infection (Høiby, 1974). Within the biofilm, the bacteria are protected against the numerous surrounding PMNs, and they exhibit a remarkable tolerance to antibiotic treatments (Donlan \& Costerton, 2002; Drenkard, 2003). The ability of bacteria to invade CF lungs in the first place is enabled by the high content of mucus and the concomitant cilia 
dysfunctionality, which result in a poor self-cleaning capacity of the CF lung (Knowles \& Boucher, 2002; Gibson et al., 2003). How the bacteria survive the encounter with the summoned PMNs prior to the formation of the protective biofilm is only partly understood, as no primary defects of PMNs from CF patients have been reported. The outcome of $P$. aeruginosa lung infections has recently been demonstrated to be at least partly dependent on quorum-sensing (QS)-regulated mechanisms (Bjarnsholt et al., 2005a; Wu et al., 2001), and the presence of QS activity during chronic $P$. aeruginosa lung infections in CF patients has been demonstrated (Storey et al., 1998; Middleton et al., 2002). QS, or cell-to-cell communication, is a regulatory mechanism by which bacteria respond to the population density (Fuqua et al., 1996). The QS systems of $P$. aeruginosa have recently been intensively investigated, and they are responsive to chemically different signal molecules: one based on $\mathrm{N}$-acylhomoserine lactone (AHL) signal molecules, and one based on 4-quinolones (4Qs). The AHL-based circuits are encoded by the Las and Rhl systems, each of which is based on LuxR and LuxI homologues. The two systems operate with specific signal molecules: $N$-3-oxododecanoyl-Lhomoserine lactone (3-oxo-C12-HSL) for the lasR-encoded receptor, and $\mathrm{N}$-butanoyl-L-homoserine lactone (C4-HSL) for the rhlR-encoded receptor. The 4Q-based system, also known as the Pseudomonas quinolone signal (PQS) system, is somewhat interspaced between the Las system and the Rhl system (McKnight et al., 2000; Pesci et al., 1999; Diggle et al., 2006). The organization and interaction of the QS system in $P$. aeruginosa has been thoroughly investigated; however, it is not yet completely understood. The Las and Rhl systems have been identified as being hierarchically ordered, with the Las system in control of the Rhl system (Pesci et al., 1997). It has been suggested, however, that the Rhl system can be switched on independently of the Las system. Diggle et al. (2003) proposed that this induction is governed by the PQS system. Intriguingly, LasR is required for the optimal production of the $4 \mathrm{Q}$ signal, whereas exogenously added PQS restores the expression of lasB in a las $R$ mutant background.

\section{METHODS}

Bacterial strains. The wild-type $P$. aeruginosa $\mathrm{PAO} 1$ used for the planktonic and biofilm in vitro experiments was obtained from the Pseudomonas Genetic Stock Center (www.pseudomonas.med.ecu. edu; strain PAO0001). This isolate has served as the DNA source for the Pseudomonas Genome Project (www.pseudomonas.com), and, subsequently, as a template for the design of the $P$. aeruginosa GeneChip (Affymetrix). The $\Delta l a s R$ rhlR and $\Delta$ lasI rhlI mutants were constructed using the knockout systems described by Beatson et al. (2002). The knockout mutants were verified by Southern blot analysis, and by screening for AHL production (quorum signals). Strains for verification of genotypes were obtained from the University of Washington, Seattle, WA, USA (see Table 1).

Production of $\boldsymbol{P}$. aeruginosa supernatants. Planktonic cultures were grown in shake flasks (180 r.p.m.) with Luria-Bertani (LB) medium at $37^{\circ} \mathrm{C}$ for $24 \mathrm{~h}$. For complementation, C4-HSL and 3-oxo-C12-HSL were added to $\Delta$ lasI rhlI mutants. Inhibition of QS was achieved by adding $12.5 \mathrm{mg}$ furanone C-30 ml $\mathrm{ml}^{-1}$ (Hentzer et al.,
2003) to the medium upon inoculation. Biofilm cultures were grown for 2 days at $37^{\circ} \mathrm{C}$ in six-well Nunc multidishes (Nunclon), each well containing $20 \mathrm{ml} \mathrm{LB}$ medium. Supernatants from planktonic and biofilm cultures were sterile-filtered through Minisart filters (16543; Sartorius), pore size $0.20 \mu \mathrm{m}$, and they were stored at $-20^{\circ} \mathrm{C}$ until use.

Biofilms for direct interaction with PMNs. Biofilms were cultivated in continuous-culture once-through flow chambers, and these were perfused with sterile $\mathrm{AB}$ trace minimal medium containing $0.3 \mathrm{mM}$ glucose, as described previously (Christensen et al., 1999; Bjarnsholt et al., 2005a).

Preparation of PMNs. Human blood samples were obtained by venous puncture from normal healthy volunteers, and collected in BD Vacutainers containing $0.129 \mathrm{M}$ sodium citrate (367704; BD Diagnostics). The PMNs were isolated by erythrocyte sedimentation and density-gradient centrifugation, as previously described (Bjarnsholt et al., 2005a).

PMN migration assay. Estimation of PMN migration against $P$. aeruginosa supernatants was carried out using Transwell trays (3415; Costar). Samples $(350 \mu \mathrm{l})$ tested were: LB medium with $10 \mathrm{nM} \mathrm{N}$ formyl-L-methionyl-L-leucyl-L-phenylalanine (fMLP) (F3506; Sigma), LB medium, and sterile filtered supernatants from PAO1, $\Delta l a s R$ rhlR, $\Delta$ lasI rhlI, and SlasI rhlI complemented with C4-HSL and 3-oxo-C12HSL. A Transwell filter (pore size, $3 \mu \mathrm{m}$ ) was inserted in the well, and $100 \mu$ isolated PMNs $\left(2.5 \times 10^{6}\right.$ cells ml $^{-1}$ in RPMI 1640 with $5 \%$ normal human $\mathrm{AB}+$ serum) was added on top of the filter. Following incubation for $30 \mathrm{~min}$ at $37^{\circ} \mathrm{C}$, the Transwell filter was removed. A $100 \mu \mathrm{l}$ volume was aspirated from the well, and added to a TruCount tube (340334; BD Biosciences) with $300 \mu \mathrm{l}$ Facslysis (349202; BD Biosciences) containing $100 \mu \mathrm{g}$ propidium iodide (PI) $\mathrm{ml}^{-1}$ (P-4170; Sigma). After incubation in the dark for a minimum of $10 \mathrm{~min}$, the samples were analysed by flow cytometry, and the number of migrated PMNs was calculated according to: migrated PMNs $=($ cells counted/beads counted $)$ (beads added/volume of cells added) $\times 10^{3} \times 0.35$. Data from each set-up were normalized by setting migration against fMLP to $100 \%$.

PMN killing by supernatants. The isolated PMNs $\left(2.5 \times 10^{6}\right.$ cells $\mathrm{ml}^{-1}$ ), and all tested sterile filtered supernatants, were equilibrated with $2.5 \mu \mathrm{g}$ PI ml${ }^{-1}$, and incubated at $37^{\circ} \mathrm{C}$ for $15 \mathrm{~min}$, before mixing $50 \mu \mathrm{l}$ isolated PMNs with $350 \mu \mathrm{l}$ sterile filtered supernatant, followed by immediate analysis of PI staining with flow cytometry.

PMN killing by biofilms. In order to inoculate PMNs into the biofilm chambers, the flow was stopped, and the flow cells were clamped off. Isolated PMNs $\left(100 \mu \mathrm{l}, 2.5 \times 10^{6} \mathrm{cells} \mathrm{ml}^{-1}\right.$, stained with $2.5 \mu \mathrm{g} \mathrm{PI} \mathrm{ml}{ }^{-1}$ ) were inoculated into each flow channel. The flow cells were incubated top down in a $37^{\circ} \mathrm{C}$ water bath, with shaking, until microscopic inspection.

Haemolysis. Normal human venous blood collected in BD Vacutainers $(100 \mu \mathrm{l})$ was mixed with $3.5 \mathrm{ml}$ sterile filtered supernatant from batch cultures of $P$. aeruginosa. After 10 min, lysis was evaluated by visual inspection.

Experimental animals. Female BALB/cj mice were purchased from M\&B Laboratory Animals at 10-11 weeks of age. The mice were of equal size, and were maintained on standard mouse chow and water ad libitum for 1 week prior to challenge. All animal experiments were authorized by the National Animal Ethics Committee, Denmark. The mouse experiments were performed as described by Pedersen et al. (1990).

\section{Isolation and staining for endobronchial PMNs}

Broncheoalveolar lavage (BAL). Exposed trachea of anaesthetized mice were canulated with a size 22 gauge catheter $\left(\mathrm{OPTIVA}^{*} 2\right.$; 
Table 1. Single-knockout mutants used in the study

Strains were obtained from the University of Washington, Seattle, WA, USA.

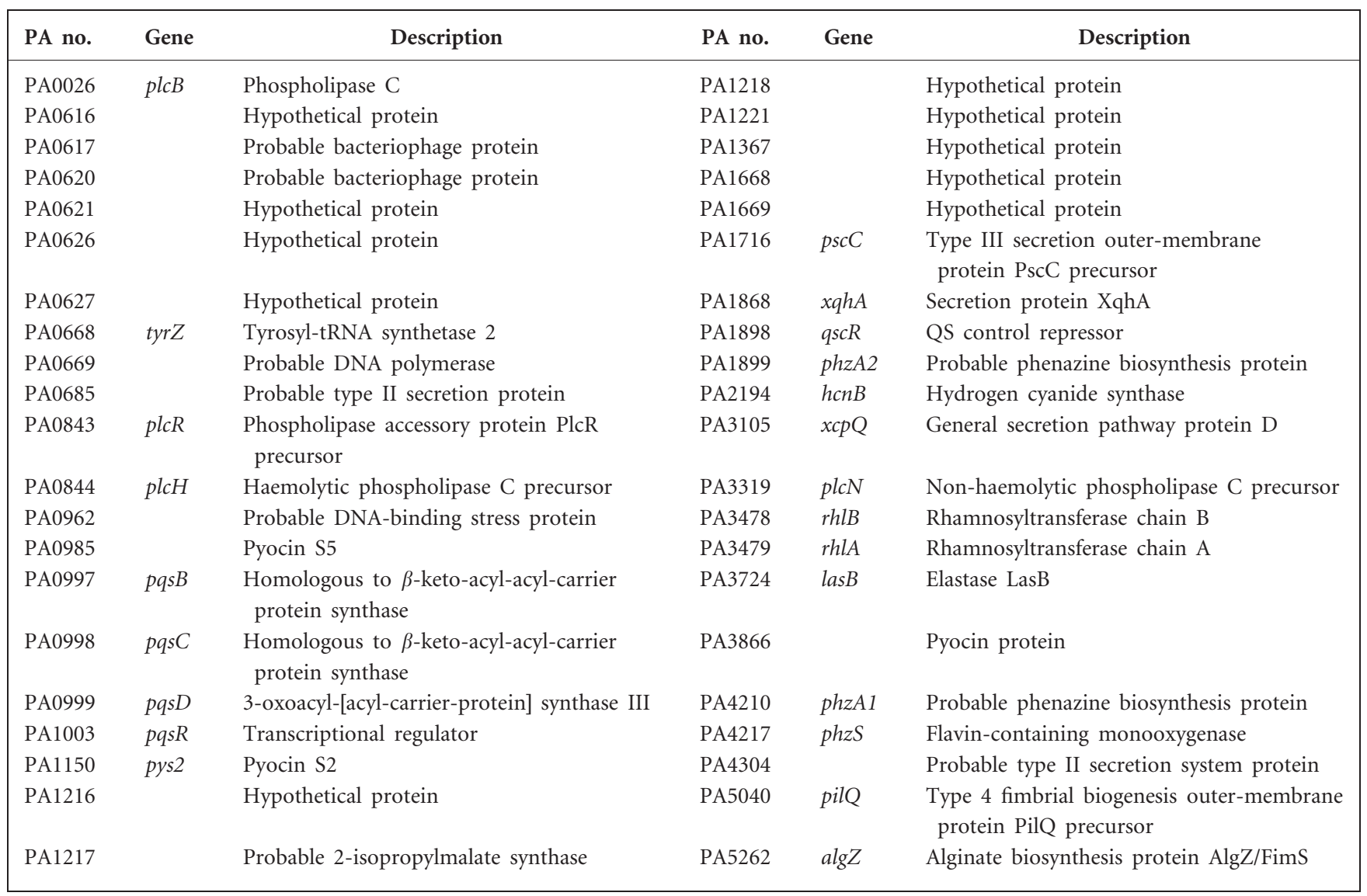

Johnson \& Johnson Medical). BAL was performed by flushing six times with $1.5 \mathrm{ml}$ ice-cold PBS without $\mathrm{Ca}^{2+}$ and $\mathrm{Mg}^{2+}$. The BAL fluid was stored on ice until staining for necrotic PMNs. The mean recovery of BAL fluid was $1.1 \mathrm{ml}$ (CV $13 \%)$.

Staining for necrotic PMNs in the BAL fluid. Necrotic and apoptotic PMNs were stained with Annexin V-FITC Apoptosis Detection Kit I (556747; BD Biosciences), according to a modification of the preparation supplied by the manufacturer. BAL fluid $(200 \mu \mathrm{l})$ was equilibrated by centrifugation with $2.5 \mathrm{ml}$ cold $1 \times$ binding buffer (BD Biosciences) at $350 \mathrm{~g}$ for $7 \mathrm{~min}$ at $5{ }^{\circ} \mathrm{C}$. To discriminate between necrotic and apoptotic PMNs, $100 \mu \mathrm{l} 1 \times$ binding buffer containing $2.5 \mu \mathrm{g}$ PI ml ${ }^{-1}$, annexin V-FITC component $(1: 40)$, and the PMN phenotypic surface marker monoclonal allophycocyanin-conjugated rat anti-Ly 6G antibody (clone RB6-8C5; BD Biosciences) (1:50), was added to the pellet, and incubated for $15 \mathrm{~min}$ at room temperature in the dark. The incubation was terminated by addition of $400 \mu \mathrm{l} 1 \times$ binding buffer, and the samples were analysed by flow cytometry.

Staining for the concentration of PMNs in the BAL fluid. A $200 \mu$ volume of BAL fluid was added to a TrueCount tube. PMNs and total leukocytes were stained by adding $20 \mu \mathrm{l}$ cold PBS containing phycoerythrin-conjugated monoclonal rat anti-mouse Ly 6G antibody (clone RB6-8C5; BD Biosciences; 1:20) and peridinin chlorophyll A protein-conjugated monoclonal rat anti-mouse CD45 antibody (clone RB6-8C5; BD Biosciences; 1:10). After incubation for $30 \mathrm{~min}$ on ice in the dark, $300 \mu \mathrm{l}$ Facslysis solution was added, and the samples were incubated for at least $10 \mathrm{~min}$ prior to flow cytometry. PMN concentration was calculated according to: cells $\mathrm{ml}^{-1}=($ cells counted/beads counted $)($ beads added/BAL fluid added) $\times 10^{3}$.

Flow cytometry. The samples were analysed using a FACSort (Becton Dickinson) equipped with a $15 \mathrm{~mW}$ argon-ion laser tuned at $488 \mathrm{~nm}$, and a red diode laser emitting at $635 \mathrm{~nm}$ for excitation. Light scatter, time, and exponentially amplified fluorescence parameters from at least 10000 events, were recorded in list mode. Necrotic PMNs were identified according to their increased PI fluorescence intensity, and their morphology was determined by light scatter. The instrument was calibrated using Calibrite beads (Becton Dickinson).

Quantitative lung bacteriology. For colony counting, the exposed lungs were isolated in $5 \mathrm{ml}$ PBS, and homogenized on ice. A serial dilution of the lung homogenate was performed, and dilutions were plated on blue agar plates (States Serum Institute), which are selective for Gram-negative bacilli.

Proteinase $\mathbf{K}$ assay. Protein degradation by proteinase $\mathrm{K}$ was performed as described by the manufacturer (Promega).

Pyocyanin assay. The pyocyanin concentration was measured as described by Essar et al. (1990).

Statistics. Data are presented as means \pm SEM; $P$ values are from Student's two-tailed unpaired $t$ tests, except for comparison of frequencies, which was done using a $\chi^{2}$ test. 


\section{RESULTS AND DISCUSSION}

\section{PMNs are unable to migrate towards the supernatant of wild-type strain $P$. aeruginosa PA01}

We have previously described the presence of a QSregulated phenotype that operates to paralyse PMNs in vitro (Bjarnsholt et al., 2005a). This inspired us to further investigate the encounter between $P$. aeruginosa and the PMNs. The PMNs are the first major phagocytes to arrive during $P$. aeruginosa lung infection, and their activity is related to the early outcome of the infection (Jensen et al., 2004). Based on our previous results, we speculated that QS-induced paralysis of the PMNs also affected migration. When freshly isolated PMNs were allowed to migrate towards sterile-filtered $P$. aeruginosa supernatants [obtained from the wild-type and the QS mutants $(\Delta l a s R$ rhlR and $\Delta$ lasI rhlI), and from $\Delta$ lasI rhlI complemented with C4-HSL and 3-oxo-C12-HSL], no migration to the wild-type and complemented supernatant was observed (Fig. 1). This effect, however, was not caused by the presence of AHL molecules, as additional experiments showed that PMNs migrated freely towards concentrations of pure AHL signal molecules 3-oxo-C12-HSL and C4-HSL increasing from 0.1 to $50 \mu \mathrm{M}$ (data not shown).

\section{PMNs are lysed by a $\boldsymbol{P}$. aeruginosa wild-type supernatant}

Elaboration of this phenomenon found that sterile-filtered supernatant from wild-type and $\Delta$ lasI rhlI mutant strains, grown in the presence of both C4-HSL and 3-oxo-C12-HSL $(10 \mu \mathrm{M})$, caused rapid damage to PMN plasma membranes, as demonstrated by increased fluorescence from supplemented PI during real-time flow cytometry (Fig. 2a).

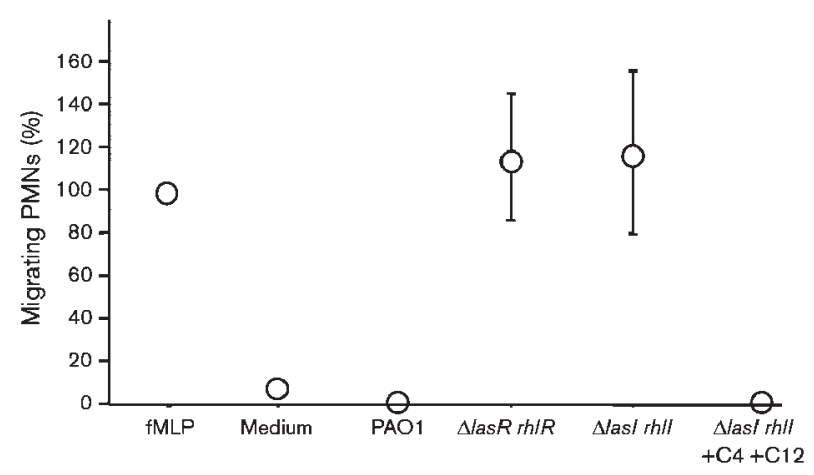

Fig. 1. Effects of $\mathrm{QS}$ on $\mathrm{PMN}$ migration. The mean $\mathrm{PMN}$ migration towards supernatants from $P$. aeruginosa with competent QS (PAO1) and deficient OS ( $\Delta / a s R$ rh/R and $\Delta / a s / r h / l)$, and a QS-deficient mutant ( $\Delta /$ as/ rhll) complemented with $\mathrm{C} 4-$ HSL (C4) and 3-oxo-C12-HSL (C12) $(10 \mu \mathrm{M})$, was calculated as the percentage of human PMNs migrated towards supernatants from $P$. aeruginosa, as compared with migration towards fMLP $(10 \mathrm{nM})$. Standard error bars are indicated $(n=6)$.
Within the first minute after the increase in PI fluorescence was recorded, the light scatter of the PMNs decreased below the threshold of detection, suggesting a rapid disintegration of the PMNs (data not shown). The rapid death and disintegration was confirmed by time-lapse recording using a combination of fluorescence and light-transmission microscopy (Fig. 2b, and supplementary movie available with the online version of this paper). Furthermore, this analysis revealed that the PMN DNA was released during disintegration. In contrast, the PMN plasma membrane remained intact when mixed with supernatants derived from $\Delta$ lasI rhlI and $\Delta l a s R$ rhlR mutants. This necrotic effect contrasts with the previously reported acceleration of PMN apoptosis caused by $P$. aeruginosa QS signal transmitter molecules (Tateda et al., 2003). Pure C4-HSL and 3-oxo$\mathrm{C} 12-\mathrm{HSL}$ at concentrations up to $50 \mu \mathrm{M}$ failed to induce rapid necrosis (data not shown).

\section{PMNs are lysed when in contact with in vitro biofilms of wild-type $\boldsymbol{P}$. aeruginosa}

To determine if the cytotoxic effect accounted for paralysis of PMNs (Bjarnsholt et al., 2005a), PMNs were incubated on an in vitro biofilm. Necrosis was observed when PMNs were incubated with QS-proficient $P$. aeruginosa biofilms grown for 4 days in flow cells (Fig. 3), but it was not observed when the PMNs were incubated on a QS-deficient biofilm grown under identical conditions. The cytotoxic effect was not specific for the PMNs, as demonstrated by the haemolytic activity of sterile-filtered supernatant from QS-competent $P$. aeruginosa cultures (Fig. 4).

\section{PMNs disappear in mice infected with wild-type $P$. aeruginosa}

To determine if the necrotic effect was restricted to in vitro settings only, a pulmonary infectious mouse model was used for verification. Previously, we established a correlation between QS deficiency and faster clearing of infecting bacteria in the mouse model (Bjarnsholt et al., 2005a). $\mathrm{BALB} / \mathrm{cj}$ mice were infected with either wild-type or QSdeficient mutant, both of which were alginate embedded. At the time points $3,6,18$ and $24 \mathrm{~h}$ post-infection, BAL fluid was obtained, after which the lungs were homogenized. Analysis of the BAL fluid by means of flow cytometry from mice infected with the wild-type $P$. aeruginosa showed a high proportion of dead PMNs in the endobronchial space, as detected by their strong PI fluorescence. In accordance with our in vitro data, significantly fewer intact PMNs (estimated by weak PI fluorescence and low annexin V staining) were found in the BAL fluids from mice infected with the wildtype $P$. aeruginosa. After 18 and $24 \mathrm{~h}$, an increased number of bacteria were observed in the lungs of mice infected with the wild-type $P$. aeruginosa (Fig. 5). This increase in the number of bacteria, however, is transient, as previous experiments have shown that the number of wild-type bacteria decreases on days 3 and 5 (Bjarnsholt et al., 2005a). Previously, we reported an elevated concentration of the PMN-recruiting interleukin MIP-2 (a murine IL-8 analogue) 


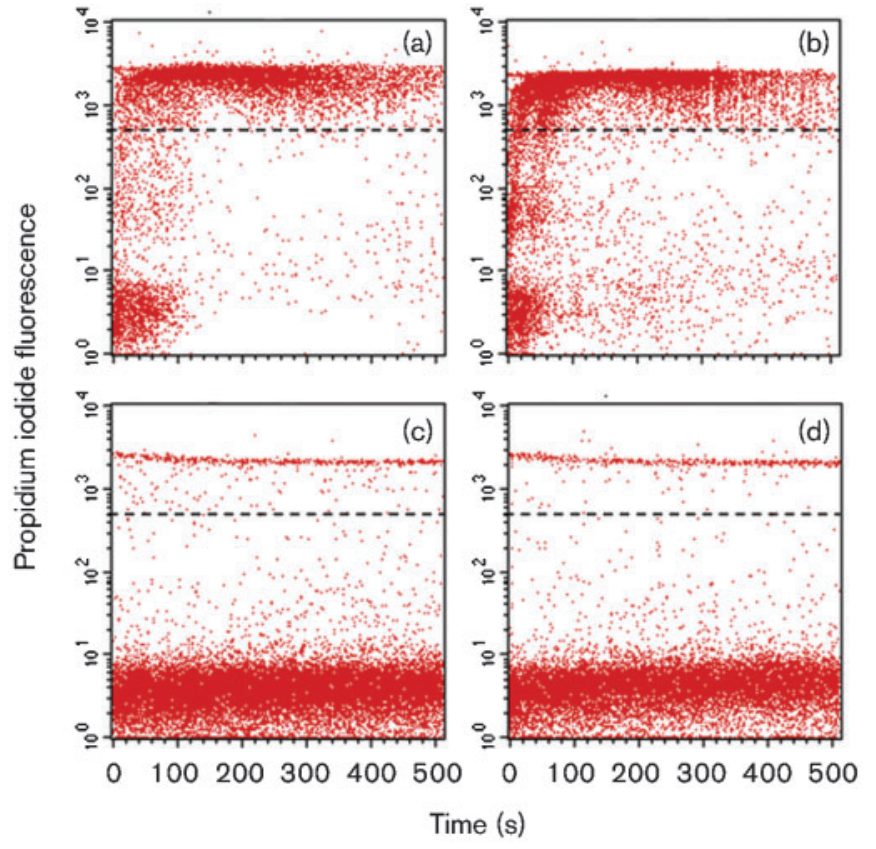

(e)

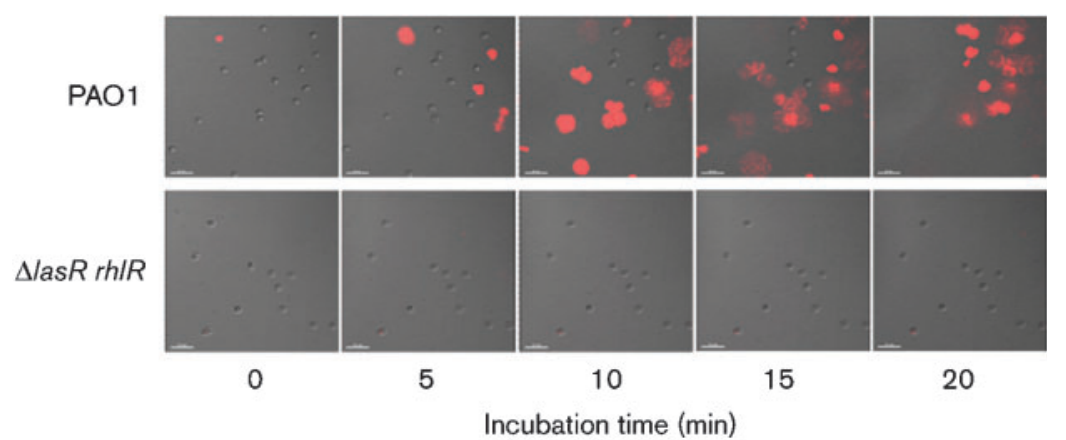

Fig. 2. QS-regulated killing of PMNs. $(a-d)$ Real-time flow cytometry of Pl-stained human PMNs mixed with sterile filtered supernatants from $P$. aeruginosa. Dashed lines represent the lower PI fluorescence intensity used for discriminating necrosis. (a) Supernatant from $P$. aeruginosa with competent OS (PAO1). (b) Supernatant from $P$. aeruginosa with deficient OS ( $\Delta / a s / \mathrm{rh} / \mathrm{l})$ complemented with C4-HSL and 3-oxoC12-HSL. (c) Supernatant from $P$. aeruginosa with deficient OS ( $\Delta / a s R \quad r h / R)$. (d) Supernatant from $P$. aeruginosa with deficient QS ( $\Delta / a s / r h / l)$. (e) Micrographs of PMN disintegration and DNA release induced by QS, and recorded by combined fluorescence and light microscopy. Human $\mathrm{PMNs}$ were mixed with sterile filtered supernatants from QS-competent (PAO1) and QS-deficient ( $\Delta / a s R$ rh/R) $P$. aeruginosa, and stained with Pl. Bars, $50 \mu \mathrm{m}$. when mice were infected with the wild-type (Bjarnsholt et al., $2005 \mathrm{~b})$. We therefore suggest that the results obtained at each time point represent snapshots of a continuous process, i.e. the PMNs are constantly being recruited to the sites of infections, but, in the wild-type situation, a substantial fraction of the incoming PMNs disintegrate due to the cytotoxic bacterial activity, which, in turn, allows the bacterial population to expand. The recruited PMNs in mice infected with the QS-deficient mutant manage to prevent an increase in the number of bacteria (Fig. 5). We suggest that this difference is caused by a QS-regulated reduction of the PMN functionality. To estimate the clinical relevance of
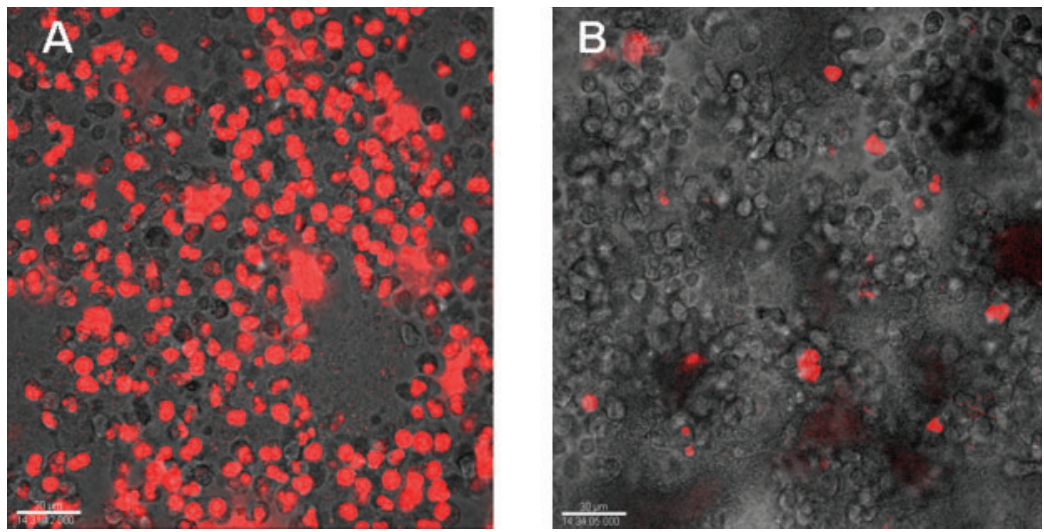

Fig. 3. QS-regulated killing of PMNs by $P$. aeruginosa biofilms visualized by combined fluorescence and light microscopy. Four-dayold biofilms, grown in continuous-culture oncethrough flow chambers, were inoculated with human PMNs, and stained with the DNA stain $\mathrm{PI}$. A greater number of PMNs became necrotic on the QS-competent PAO1 biofilm (A) compared with the QS-mutant ( $\Delta / a s R$ rh/R) biofilm (B). Bars, $30 \mu \mathrm{m}$. 


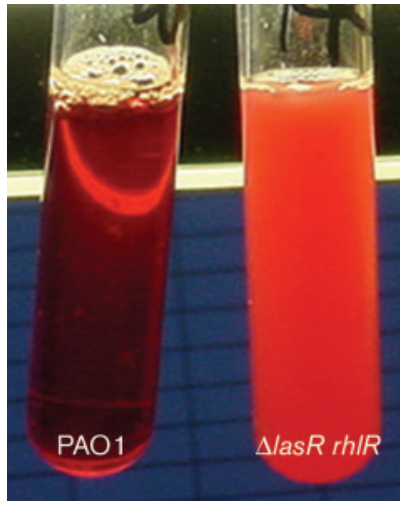

Fig. 4. QS-regulated haemolysis of whole blood. Human peripheral blood was mixed with sterile filtered supernatants from $P$. aeruginosa. Haemolysis was only induced with supernatant from QS-competent $P$. aeruginosa (PAO1).

cytotoxic metabolites from $P$. aeruginosa, sputum from CF patients chronically infected with and without $P$. aeruginosa was compared. Of the sputum samples from patients with $P$. aeruginosa infections, 9 of 13 induced rapid PMN necrosis in our in vitro assay, whereas 0 of 3 samples from patients without $P$. aeruginosa induced $\mathrm{PMN}$ necrosis $(P \leqslant 0.03)$.

\section{The QS inhibitor furanone C-30 blocks cytotoxicity in vitro}

Since the effect of QS blockers, such as furanone C-30, results in accelerated clearance of $P$. aeruginosa in the pulmonary mouse model (Hentzer et al., 2003), it was of interest to determine if the induction of PMN necrosis by treating a growing batch culture with furanone C-30 could be blocked. We observed that furanone-C-30-treated cultures of the wild-type, and a $\Delta$ las I rhlI mutant grown in the presence of exogenously added AHL signal molecules, completely blocked development of both the PMN necrotic (Fig. 6) and the haemolytic effect (not shown).

\section{Identification of the QS-regulated cytotoxin}

Based upon these results, we conclude that the QS-regulated necrotic PMN factor is an extracellular product that causes PMN malfunction both in vivo and in vitro. As for $P$. aeruginosa, a minimum of 172 genes are QS regulated, and many of these are only annotated as hypothetical proteins (Hentzer et al., 2003). Several of the QS-regulated genes encode known virulence factors, including proteins, lipids and secondary metabolites (Hentzer et al., 2003; Wagner et al., 2003; Schuster et al., 2003). In addition, several virulence factors with potentially necrotic capacity are not QS regulated, and these include exotoxin A and pepA (exoU is not present in PAO1). Furthermore, the type III secretion apparatus is dependent on cell-cell contact for delivery of toxins; in contrast, the effect we describe in the present report is not dependent on this process. AHLs have been described to induce apoptosis in macrophages and PMNs (Tateda et al., 2003); however, we were unable to replicate the fast killing of the PMNs by treatment with pure AHL signal molecules (data not shown). To identify the compound responsible for the rapid necrotic killing, the supernatant of the wild-type was examined as described below. The active component was identified as rhamnolipid B, which belongs to a class of well-known biosurfactants from $P$. aeruginosa. To verify this, single-knockout mutants were investigated, including rhamnolipid, hydrogen cyanide, elastase and phospholipase C; for a complete list of strains tested refer to Table 1. Supernatants of these singleknockout mutants suggested that rhamnolipids were responsible for the necrotic activity identified by flow cytometry of PI-stained PMNs. Induction of PMN necrosis was also found to be independent of the pyocyanin content (data not shown). P. aeruginosa produces quinolones (PQS) as another part of its QS apparatus, and these molecules have been shown to induce apoptosis and reduce T-cell proliferation (Calfee et al., 2005; Pritchard, 2006). No necrotic effect was observed in the PMNs upon direct addition of up to $100 \mu \mathrm{M}$ PQS (data not shown). We did, however, find that $\Delta p q s B, \Delta p q s C, \Delta p q s D$ and $\Delta p q s R$ mutants did not produce the necrotic effect, indicating that the PQS system is involved in the regulation of the production of the compound(s) causing PMN necrosis.

The principal necrotic metabolite was identified from supernatants as $2-O-\alpha$-L-rhamnopyranosyl- $\alpha$-L-rhamnopyranosyl- $\beta$-hydroxydecanoyl- $\beta$-hydroxydecanoic acid, which is also known as rhamnolipid B. Supernatants of outgrown $P$. aeruginosa $\mathrm{PAO} 1$ batch cultures were found to contain 100-200 $\mu \mathrm{g}$ rhamnolipid $\mathrm{B} \mathrm{ml}^{-1}$, whereas no rhamnolipids
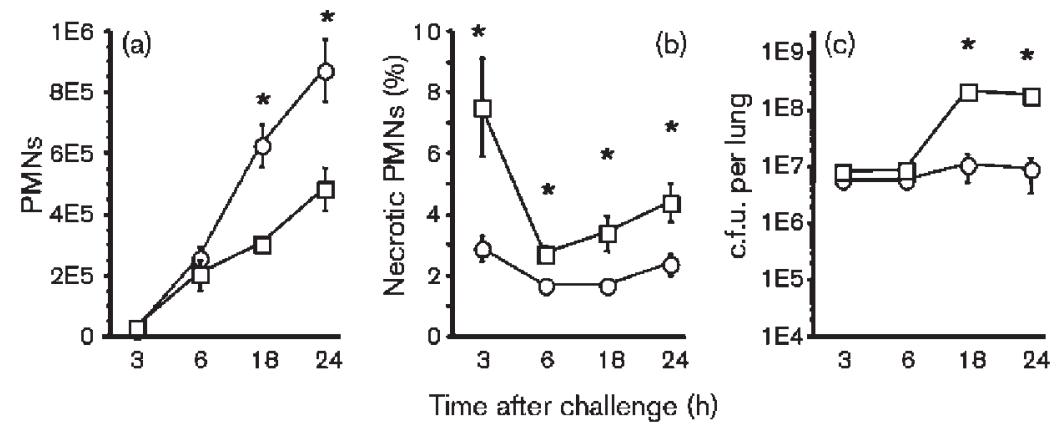

Fig. 5. Significance of as during $P$. aeruginosa lung infection. $B A L B / c j$ mice were inoculated with alginate-embedded OScompetent (PAO1, $\square$ ) or OS-deficient $(\Delta / a s R$ rhIR, O) $P$. aeruginosa in the left lung, and sampled at $3,6,18$ and $24 \mathrm{~h}$. (a) Mean number of intact $\mathrm{PMNs}$ in the BAL fluid. (b) Mean percentage of necrotic PMNs in the BAL fluid. (c) Mean number of c.f.u. in the lungs. Standard error bars are indicated. ${ }^{*} P<0.01, n=10$; unpaired $t$ test. 


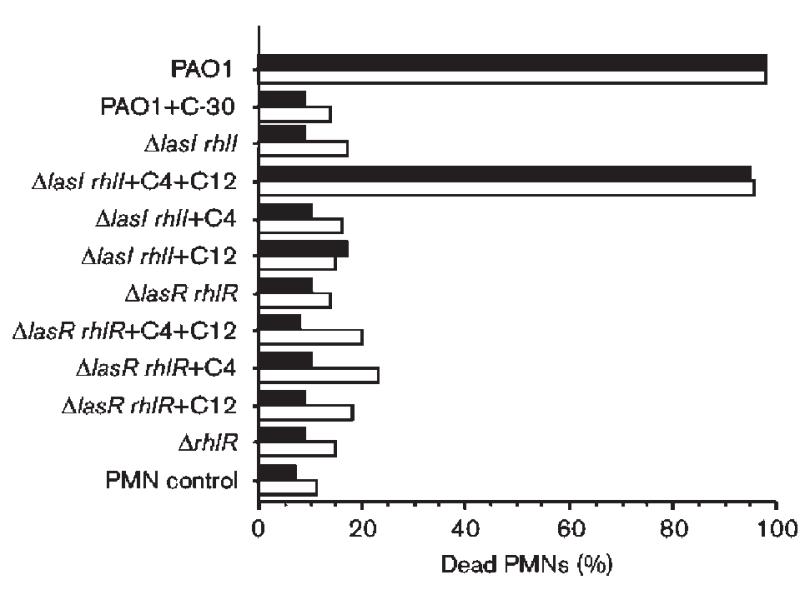

Fig. 6. Inhibition and complementation of the QS-regulated fast killing of PMNs. The contents of dead PMNs were analysed by flow cytometry of human PMNs stained with PI, and mixed with sterile filtered supernatants from cultures of $P$. aeruginosa for 5 (black bars) and $10 \mathrm{~min}$ (white bars). Inhibition of aS was done by adding synthetic furanone C-30 to PAO1 during culture, and with double-knockout ( $\Delta / a s R$ rh/R and $\Delta / a s / r h / l)$ and single-knockout $(\Delta / a s R)$ mutations. Complementation was performed by growing $\Delta / a s / r h / l$ in the presence of C4-HSL (C4) and/or 3-oxo-C12-HSL (C12). The bars show representative values from several experiments.

were detected in $\triangle r h l A$ mutants and a $\Delta l a s R$ rhlR mutant. In addition, PQS mutants were found to produce far less rhamnolipid B than the wild-type. Incubating PMNs with wild-type supernatant containing approximately $100 \mu \mathrm{g}$ rhamnolipid $\mathrm{B} \mathrm{ml}^{-1}$ induced necrosis as fast as incubation with $100 \mu \mathrm{g}$ purified rhamnolipid $\mathrm{B} \mathrm{ml}^{-1}$. Earlier investigations support our finding, as rhamnolipids are known to lyse PMNs (Shryock et al., 1984), erythrocytes (Johnson \& Boese-Marrazzo, 1980), and monocyte-derived macrophages (McClure \& Schiller, 1992).

\section{Rhamnolipid isolation and identification}

Sterile filtered culture supernatant from PAO1 (3 l) was extracted three times in ethyl acetate $(3 \times 21)$, and the ethyl acetate was removed under vacuum to yield $1.07 \mathrm{~g}$ yellow solid material. This was adsorbed to celite, and applied to a $10 \mathrm{~g}$ isolute DIOL column pre-equilibrated with $100 \%$ heptane. Fractions were eluted as follows: two fractions of $50 \%$ dichloromethane (DCM) in heptane; two fractions of $100 \%$ DCM; 20, 30, 40, 50, 60 and $80 \%$ ethyl acetate in DCM; $100 \%$ ethyl acetate; $10 \%$ methanol in ethyl acetate; and, finally, two $100 \%$ methanol washes. All fractions were $12 \mathrm{ml}$, except for the last methanol wash, which was $50 \mathrm{ml}$. PMN necrotic activity was seen in the last three fractions; these fractions were combined, and then further fractionated on a $20 \mathrm{~g}$ StrataX $\mathrm{C}_{18}$ column. Elution was with a stepped acetonitrile/water gradient starting at $100 \%$ water, increasing to $100 \%$ acetonitrile in $10 \%$ steps, skipping 10 and $90 \%$ acetonitrile fractions, and collecting $50 \mathrm{ml}$ per fraction. The primary PMN necrotic activity was detected in the seventh fraction ( $80 \%$ acetonitrile). LC-MS showed a molecular mass of $651.3881 \mathrm{~g} \mathrm{~mol}^{-1}$ (Fig. 7), corresponding to a molecular formula of $\mathrm{C}_{32} \mathrm{H}_{58} \mathrm{O}_{13}(4 \mathrm{dbe})$. Other ions were observed at $359.3,505.3,668.4$ and $673.4 \mathrm{~g} \mathrm{~mol}^{-1}$,

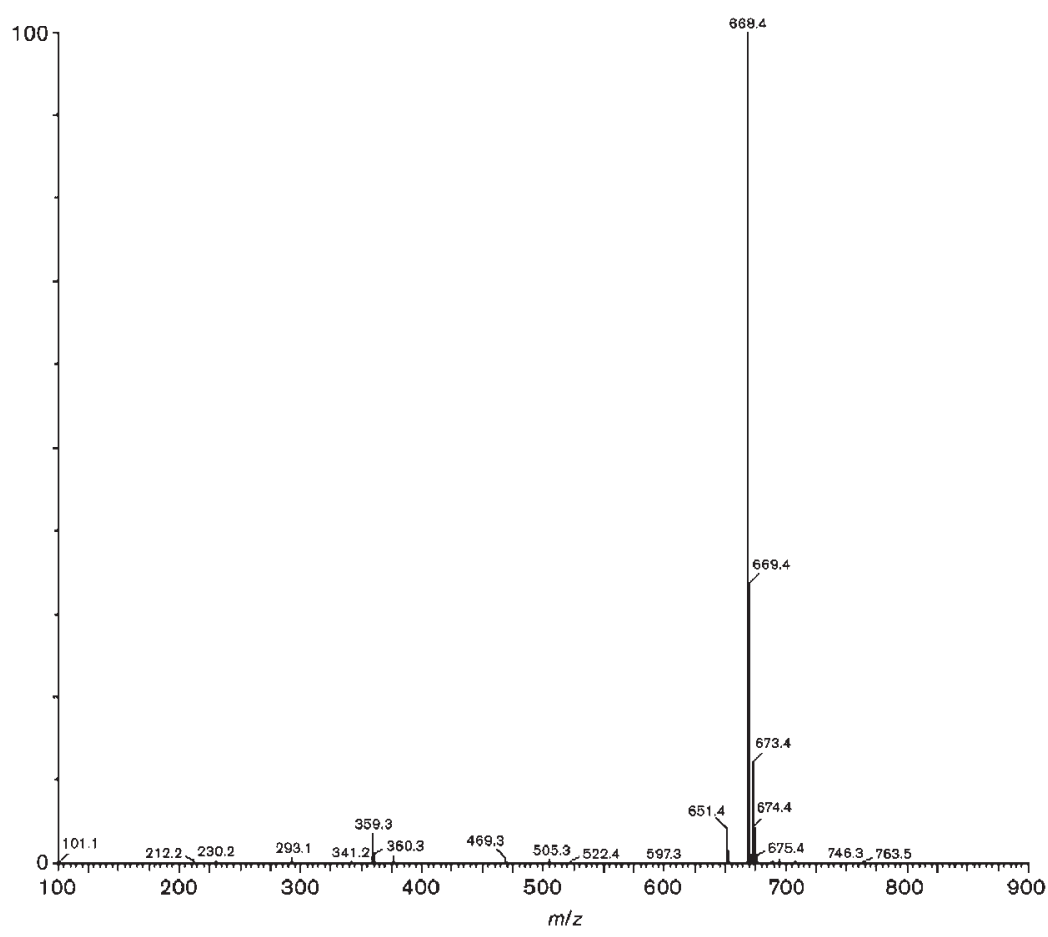

http://mic.sgmjournals.org
Fig. 7. Mass spectrum of rhamnolipid B purified from supernatant of $P$. aeruginosa PAO1. 


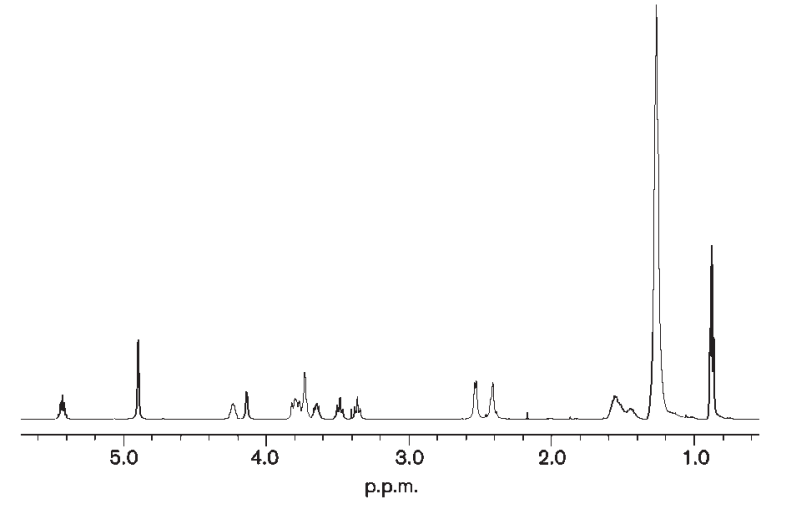

Fig. 8. ${ }^{1} \mathrm{H}$ NMR spectrum of rhamnolipid $B$ purified from supernatant of $P$. aeruginosa PAO1. The spectrum was referenced to $\mathrm{CDCl}_{3}$ at $\delta_{\mathrm{H}} 7.26$, and obtained on a Varian Inova operating at $500 \mathrm{MHz}$.

corresponding to $[\mathrm{M}+\mathrm{H}-2 \times \text { rhamnose }]^{+},[\mathrm{M}+\mathrm{H}$-rhamnose $]^{+},\left[\mathrm{M}+\mathrm{NH}_{3}\right]^{+}$and $[\mathrm{M}+\mathrm{Na}]^{+}$, respectively.

The ${ }^{1} \mathrm{H}$ NMR spectrum (Fig. 8) showed two anomeric signals, several glycosidic proton signals, and a large number of aliphatic signals. The ${ }^{13} \mathrm{C}$ NMR spectrum indicated the presence of two carbonyl ester carbons, two anomeric carbons, several oxygenated carbons, with the remaining signals corresponding to aliphatic and methyl carbons. Analysis of the 2D NMR spectra (Table 2) showed two rhamnose and two fatty acid units, suggesting that the molecule was a rhamnolipid, which is a class of compounds well known from $P$. aeruginosa. Extremely good agreement was seen between the experimental NMR data and that published by Sim et al. (1997) for the compound 2-O- $\alpha-\mathrm{L}-$ rhamnopyranosyl- $\alpha$-L-rhamnopyranosyl- $\beta$-hydroxydecanoyl$\beta$-hydroxydecanoic acid, or rhamnolipid B.

\section{Conclusion}

Functional QS systems are present in the vast majority of $P$. aeruginosa isolates from the urinary tract, the lower respiratory tract and wound infections (Schaber et al., 2004), and the presence of functional QS systems significantly delays clearing of the bacteria in experimental studies of $P$. aeruginosa pulmonary infections and thermal wounds $(\mathrm{Wu}$ et al., 2001; Bjarnsholt et al., 2005a; Rumbaugh et al., 1999). Interestingly, $P$. aeruginosa frequently loses the ability to produce the long-chain signal molecule over time (Heurlier et al., 2006), suggesting a pivotal role of QS during the initial stages of infection. It is now widely accepted that $P$. aeruginosa adapts to the lung environment, to the point of evolving several subpopulations throughout the lung. One of the main adaptations involves production of alginate,

Table 2. NMR data of rhamnolipid B

Data were acquired on a Varian Inova machine at $500 \mathrm{MHz}$ for ${ }^{1} \mathrm{H}$, and $300 \mathrm{MHz}$ for ${ }^{13} \mathrm{C}$. Spectra were referenced to $\mathrm{CDCl}_{3}$ at $\delta_{\mathrm{H}}$ 7.26. The shifts for the atoms numbered from 2 to 7 , and from 12 to 17 , were not included in the table as they all overlapped in the region of $\delta_{\mathrm{H}} 1.2-1.5$ for proton, and $\delta_{\mathrm{C}}$ 25-30 for carbon. COSY, correlated spectroscopy; HMBC, heteronuclear multiple-bond correlation.

\begin{tabular}{|c|c|c|c|c|}
\hline Number & $\delta_{\mathrm{H}}$, mult., $\left(J_{\mathrm{HH}} \mathrm{Hz}\right)$ & $\cos Y$ & ${ }^{13} \mathrm{C}$ & НМВС \\
\hline 1 & $0.87, \mathrm{~d}$ & 1.29 & 13.9 & - \\
\hline 8 & 4.23 , sept & $2.41,1.45$ & 70.9 & - \\
\hline 9 & $2.41, \mathrm{~m}$ & 4.23 & 39.4 & $29.1,24.6$ \\
\hline 10 & - & - & 171.0 & - \\
\hline 11 & $0.86, \mathrm{~d}$ & 1.29 & 13.9 & - \\
\hline 18 & $5.43, \mathrm{p},(6.2)$ & $1.56,2.53$ & 70.2 & $24.9,171.3,173.6$ \\
\hline 19 & $2.53, \mathrm{~d},(5.9)$ & 5.43 & 39.1 & $171.3,71.2$ \\
\hline 20 & - & - & 173.0 & - \\
\hline $1^{\prime}$ & 4.89 , br s & 4.13 & 94.2 & $68.0,70.1,71.5,79.7$ \\
\hline $2^{\prime}$ & 4.13 , br s & $4.89,3.77$ & 70.3 & $71.0,72.3$ \\
\hline $3^{\prime}$ & $3.77, \mathrm{dd},(2.4,10.3)$ & $4.13,3.48$ & 71.1 & - \\
\hline $4^{\prime}$ & $3.48, \mathrm{t},(9.1)$ & $3.77,3.73$ & 72.2 & $70.5,68.0,17.7$ \\
\hline $5^{\prime}$ & 3.73 , br $\mathrm{q},(7.0)$ & $3.48,1.26$ & 68.5 & 71.0 \\
\hline $6^{\prime}$ & $1.27, \mathrm{~d},(6)$ & $3.73,1.45$ & 17.4 & - \\
\hline $7^{\prime}$ & 4.89, br s & 3.73 & 102.1 & $68.0,70.1,71.5,79.7$ \\
\hline $8^{\prime}$ & $3.73, \mathrm{~m}$ & $4.89,3.80$ & 79.4 & $102.5,73.4$ \\
\hline $9^{\prime}$ & $3.80, \mathrm{dd},(1.8,9.6)$ & $3.36,3.73$ & 70.3 & - \\
\hline $10^{\prime}$ & $3.36, \mathrm{t},(9.4)$ & $3.80,3.65$ & 73.2 & $173.6,70.5$ \\
\hline $11^{\prime}$ & $3.65, \mathrm{dt},(5.6,14)$ & $3.36,1.27$ & 67.5 & $71.0,68.7,17.7$ \\
\hline $12^{\prime}$ & $1.26, \mathrm{~d},(6)$ & $3.65,1.56$ & 24.9 & - \\
\hline
\end{tabular}


which is believed to represent a long-term defence mechanism. In contrast, the killing of the PMNs may be one of the mechanisms that facilitates establishment of early $P$. aeruginosa lung infections. In support of this model, a higher number of necrotic PMNs has been detected in CF lungs infected with $P$. aeruginosa, as compared with other bacterial infections (Watt et al., 2005). In addition, CF lungs chronically infected with $P$. aeruginosa contain high amounts of extracellular DNA, which is probably derived from necrotic PMNs (Lethem et al., 1990; Shah et al., 1996). The ability of $P$. aeruginosa to defend itself aggressively against PMNs by production of rhamnolipids may be a prerequisite for the development of the chronic infection with $P$. aeruginosa. As rhamnolipids have been detected in sputum from CF patients with chronic $P$. aeruginosa lung infections (Kownatzki et al., 1987), and nonmucoid clinical isolates have been shown to produce more haemolytically active rhamnolipids than mucoid isolates (McClure \& Schiller, 1992), we believe that our observations add significantly to understanding the basis of how QS regulates the phenotype of $P$. aeruginosa for successful establishment of chronic infections, as seen in the CF lung. Several characteristics of the infected CF lungs are currently considered as being able to promote the persistence of $P$. aeruginosa. In this context, our demonstration of QS-regulated secretion of cytotoxic amounts of rhamnolipids by $P$. aeruginosa may add significantly to understanding the establishment and persistence of the infection: impairment of neutrophil migration caused by the viscous mucus has been proposed as a mechanism for decreased bactericidal activity (Matsui et al., 2005). Baltimore et al. (1989), however, have clearly shown that the neutrophils are able to accumulate very close to the microcolonies of $P$. aeruginosa in the CF lung. Despite this massive PMN accumulation, the microcolonies persist, suggesting another mechanism by which the bacteria impair the nearby neutrophils. Impairment of the neutrophils has also been proposed to be caused by QS-regulated proteases cleaving surface receptors on the neutrophils (Kharazmi et al., 1984a, b); however, neutralizing antibodies against these proteases are present in the lungs of chronically infected CF patients (Doring et al., 1985). We found that QS-induced PMN necrosis was not caused by proteins. In addition, the formation of biofilm has been recognized as a mechanism for protection against phagocytosis (Donlan \& Costerton, 2002); however, we previously demonstrated that biofilm-induced protection against phagocytosis by neutrophils is dependent on QS (Bjarnsholt et al., 2005a). Further work is necessary to elucidate the role and consequences of the presence of QS-regulated rhamnolipds during the colonization and infection of CF lungs.

\section{ACKNOWLEDGEMENTS}

We would like to thank the nurses at the Danish Cystic Fibrosis Center for collecting samples. M.G. received financial support from the Danish Research Council FTP-supported 'A new approach to the control of microbial activity', the Biomedical Consortium 'Biomed', and the German Mukoviszidose e.v. N.H. received financial support from the Danish Research Agency (22-02-0203) and the Research Council of Rigshospitalet (410-005). C. M. received financial support from the Toyota Foundation.

\section{REFERENCES}

Baltimore, R. S., Christie, C. D. \& Smith, G. J. (1989). Immunohistopathologic localization of Pseudomonas aeruginosa in lungs from patients with cystic fibrosis. Implications for the pathogenesis of progressive lung deterioration. Am Rev Respir Dis 140, 1650-1661.

Beatson, S. A., Whitchurch, C. B., Semmler, A. B. \& Mattick, J. S. (2002). Quorum sensing is not required for twitching motility in Pseudomonas aeruginosa. J Bacteriol 184, 3598-3604.

Bjarnsholt, T., Jensen, P. O., Burmolle, M., Hentzer, M., Haagensen, J. A. J., Hougen, H. P., Calum, H., Madsen, K. G., Moser, C. \& other authors (2005a). Pseudomonas aeruginosa tolerance to tobramycin, hydrogen peroxide and polymorphonuclear leukocytes is quorumsensing dependent. Microbiology 151, 373-383.

Bjarnsholt, T., Jensen, P. O., Rasmussen, T. B., Christophersen, L., Calum, H., Hentzer, M., Hougen, H. P., Rygaard, J., Moser, C. \& other authors (2005b). Garlic blocks quorum sensing and promotes rapid clearing of pulmonary Pseudomonas aeruginosa infections. Microbiology 151, 3873-3880.

Calfee, M. W., Shelton, J. G., McCubrey, J. A. \& Pesci, E. C. (2005). Solubility and bioactivity of the Pseudomonas quinolone signal are increased by a Pseudomonas aeruginosa-produced surfactant. Infect Immun 73, 878-882.

Christensen, B. B., Sternberg, C., Andersen, J. B., Palmer, R. J., Jr, Nielsen, A. T., Givskov, M. \& Molin, S. (1999). Molecular tools for study of biofilm physiology. Methods Enzymol 310, 20-42.

Diggle, S. P., Winzer, K., Chhabra, S. R., Worrall, K. E., Camara, M. \& Williams, P. (2003). The Pseudomonas aeruginosa quinolone signal molecule overcomes the cell density-dependency of the quorum sensing hierarchy, regulates $r h l$-dependent genes at the onset of stationary phase and can be produced in the absence of LasR. Mol Microbiol 50, 29-43.

Diggle, S. P., Cornelis, P., Williams, P. \& Camara, M. (2006). 4-Quinolone signalling in Pseudomonas aeruginosa: old molecules, new perspectives. Int J Med Microbiol 296, 83-91.

Donlan, R. M. \& Costerton, J. W. (2002). Biofilms: survival mechanisms of clinically relevant microorganisms. Clin Microbiol Rev 15, 167-193.

Doring, G., Goldstein, W., Roll, A., Schiotz, P. O., Høiby, N. \& Botzenhart, K. (1985). Role of Pseudomonas aeruginosa exoenzymes in lung infections of patients with cystic fibrosis. Infect Immun 49, 557-562.

Drenkard, E. (2003). Antimicrobial resistance of Pseudomonas aeruginosa biofilms. Microbes Infect 5, 1213-1219.

Essar, D. W., Eberly, L., Hadero, A. \& Crawford, I. P. (1990). Identification and characterization of genes for a second anthranilate synthase in Pseudomonas aeruginosa: interchangeability of the two anthranilate synthases and evolutionary implications. J Bacteriol 172, 884-900.

Fuqua, C., Winans, S. C. \& Greenberg, E. P. (1996). Census and consensus in bacterial ecosystems: the LuxR-LuxI family of quorumsensing transcriptional regulators. Annu Rev Microbiol 50, 727-751.

Gibson, R. L., Burns, J. L. \& Ramsey, B. W. (2003). Pathophysiology and management of pulmonary infections in cystic fibrosis. Am J Respir Crit Care Med 168, 918-951.

Hentzer, M., Wu, H., Andersen, J. B., Riedel, K., Rasmussen, T. B., Bagge, N., Kumar, N., Schembri, M. A., Song, Z. \& other authors 
(2003). Attenuation of Pseudomonas aeruginosa virulence by quorum sensing inhibitors. EMBO J 22, 3803-3815.

Heurlier, K., Denervaud, V. \& Haas, D. (2006). Impact of quorum sensing on fitness of Pseudomonas aeruginosa. Int J Med Microbiol 296, 93-102.

Høiby, N. (1974). Pseudomonas aeruginosa infection in cystic fibrosis. Relationship between mucoid strains of Pseudomonas aeruginosa and the humoral immune response. Acta Pathol Microbiol Scand [B] Microbiol Immunol 82, 551-558.

Jensen, P. Ø., Moser, C., Kobayashi, O., Hougen, H. P., Kharazmi, A. \& Høiby, N. (2004). Faster activation of polymorphonuclear neutrophils in resistant mice during early innate response to Pseudomonas aeruginosa lung infection. Clin Exp Immunol 137, 478-485.

Johansen, H. K. \& Høiby, N. (1992). Seasonal onset of initial colonisation and chronic infection with Pseudomonas aeruginosa in patients with cystic fibrosis in Denmark. Thorax 47, 109-111.

Johnson, M. K. \& Boese-Marrazzo, D. (1980). Production and properties of heat-stable extracellular hemolysin from Pseudomonas aeruginosa. Infect Immun 29, 1028-1033.

Kharazmi, A., Doring, G., Høiby, N. \& Valerius, N. H. (1984a). Interaction of Pseudomonas aeruginosa alkaline protease and elastase with human polymorphonuclear leukocytes in vitro. Infect Immun 43, 161-165.

Kharazmi, A., Høiby, N., Doring, G. \& Valerius, N. H. (1984b). Pseudomonas aeruginosa exoproteases inhibit human neutrophil chemiluminescence. Infect Immun 44, 587-591.

Knowles, M. R. \& Boucher, R. C. (2002). Mucus clearance as a primary innate defense mechanism for mammalian airways. J Clin Invest 109, 571-577.

Koch, C. \& Høiby, N. (1993). Pathogenesis of cystic fibrosis. Lancet 341, 1065-1069.

Kownatzki, R., Tummler, B. \& Doring, G. (1987). Rhamnolipid of Pseudomonas aeruginosa in sputum of cystic fibrosis patients. Lancet 1, 1026-1027.

Lethem, M. I., James, S. L., Marriott, C. \& Burke, J. F. (1990). The origin of DNA associated with mucus glycoproteins in cystic fibrosis sputum. Eur Respir J 3, 19-23.

Mathee, K., Ciofu, O., Sternberg, C., Lindum, P. W., Campbell, J. I., Jensen, P., Johnsen, A. H., Givskov, M. M., Ohman, D. E. \& other authors (1999). Mucoid conversion of Pseudomonas aeruginosa by hydrogen peroxide: a mechanism for virulence activation in the cystic fibrosis lung. Microbiology 145, 1349-1357.

Matsui, H., Verghese, M. W., Kesimer, M., Schwab, U. E., Randell, S. H., Sheehan, J. K., Grubb, B. R. \& Boucher, R. C. (2005). Reduced three-dimensional motility in dehydrated airway mucus prevents neutrophil capture and killing bacteria on airway epithelial surfaces. J Immunol 175, 1090-1099.

McClure, C. D. \& Schiller, N. L. (1992). Effects of Pseudomonas aeruginosa rhamnolipids on human monocyte-derived macrophages. J Leukoc Biol 51, 97-102.

McKnight, S. L., Iglewski, B. H. \& Pesci, E. C. (2000). The Pseudomonas quinolone signal regulates $r h l$ quorum sensing in Pseudomonas aeruginosa. J Bacteriol 182, 2702-2708.

Middleton, B., Rodgers, H. C., Camara, M., Knox, A. J., Williams, P. \& Hardman, A. (2002). Direct detection of $N$-acylhomoserine lactones in cystic fibrosis sputum. FEMS Microbiol Lett 207, 1-7.

Pedersen, S. S., Shand, G. H., Hansen, B. L. \& Hansen, G. N. (1990). Induction of experimental chronic Pseudomonas aeruginosa lung infection with $P$. aeruginosa entrapped in alginate microspheres. APMIS 98, 203-211.

Pesci, E. C., Pearson, J. P., Seed, P. C. \& Iglewski, B. H. (1997). Regulation of las and rhl quorum sensing in Pseudomonas aeruginosa. J Bacteriol 179, 3127-3132.

Pesci, E. C., Milbank, J. B., Pearson, J. P., McKnight, S., Kende, A. S., Greenberg, E. P. \& Iglewski, B. H. (1999). Quinolone signaling in the cell-to-cell communication system of Pseudomonas aeruginosa. Proc Natl Acad Sci U S A 96, 11229-11234.

Pritchard, D. I. (2006). Immune modulation by Pseudomonas aeruginosa quorum-sensing signal molecules. Int J Med Microbiol 296, 111-116.

Rumbaugh, K. P., Griswold, J. A., Iglewski, B. H. \& Hamood, A. N. (1999). Contribution of quorum sensing to the virulence of Pseudomonas aeruginosa in burn wound infections. Infect Immun 67, 58545862.

Schaber, J. A., Carty, N. L., McDonald, N. A., Graham, E. D., Cheluvappa, R., Griswold, J. A. \& Hamood, A. N. (2004). Analysis of quorum sensing-deficient clinical isolates of Pseudomonas aeruginosa. $J$ Med Microbiol 53, 841-853.

Schuster, M., Lostroh, C. P., Ogi, T. \& Greenberg, E. P. (2003). Identification, timing, and signal specificity of Pseudomonas aeruginosa quorum-controlled genes: a transcriptome analysis. J Bacteriol 185, 2066-2079.

Shah, P. L., Scott, S. F., Knight, R. A. \& Hodson, M. E. (1996). The effects of recombinant human DNase on neutrophil elastase activity and interleukin-8 levels in the sputum of patients with cystic fibrosis. Eur Respir J 9, 531-534.

Shryock, T., Silver, A. S., Baschbach, M. W. \& Kramer, J. C. (1984). Effect of Pseudomonas aeruginosa rhamnolipid on human neutrophil migration. Curr Microbiol 10, 323-328.

Sim, L., Ward, O. P. \& Li, Z. Y. (1997). Production and characterisation of a biosurfactant isolated from Pseudomonas aeruginosa UW-1. J Ind Microbiol Biotechnol 19, 232-238.

Storey, D. G., Ujack, E. E., Rabin, H. R. \& Mitchell, I. (1998). Pseudomonas aeruginosa lasR transcription correlates with the transcription of las $A$, las $B$, and tox $A$ in chronic lung infections associated with cystic fibrosis. Infect Immun 66, 2521-2528.

Tateda, K., Ishii, Y., Horikawa, M., Matsumoto, T., Miyairi, S., Pechere, J. C., Standiford, T. J., Ishiguro, M. \& Yamaguchi, K. (2003). The Pseudomonas aeruginosa autoinducer $N$-3-oxododecanoyl homoserine lactone accelerates apoptosis in macrophages and neutrophils. Infect Immun 71, 5785-5793.

Wagner, V. E., Bushnell, D., Passador, L., Brooks, A. I. \& Iglewski, B. H. (2003). Microarray analysis of Pseudomonas aeruginosa quorumsensing regulons: effects of growth phase and environment. J Bacteriol 185, 2080-2095.

Watt, A. P., Courtney, J., Moore, J., Ennis, M. \& Elborn, J. S. (2005). Neutrophil cell death, activation and bacterial infection in cystic fibrosis. Thorax 60, 659-664.

Worlitzsch, D., Tarran, R., Ulrich, M., Schwab, U., Cekiki, A., Meyer, K. C., Birrer, P., Bellon, G., Berger, J. \& other authors (2002). Effects of reduced mucus oxygen concentration in airway Pseudomonas infections of cystic fibrosis patients. J Clin Invest 109, 317-325.

Wu, H., Song, Z., Givskov, M., Doring, G., Worlitzsch, D., Mathee, K., Rygaard, J. \& Høiby, N. (2001). Pseudomonas aeruginosa mutations in lasI and rhlI quorum sensing systems result in milder chronic lung infection. Microbiology 147, 1105-1113.

Edited by: P. Cornelis 